\title{
A rare cause of esophagitis with crystal deposition
}

\author{
Vinaya Gaduputi MD, Hassan Tariq MD, Ariyo Ihimoyan MD
}

\section{CASE PRESENTATION}

A 77-year-old woman with poorly differentiated signet ring cell gastric adenocarcinoma (T4aN1M0, Stage III-B), for which she underwent partial gastrectomy and received chemotherapy with leucovorin and 5-flourouracil (5-FU), presented to hospital for elective endoscopic evaluation of an abnormal computed tomography (CT) scan that revealed thickening in the proximal stomach. Her medical comorbidities included type 2 diabetes mellitus, benign essential hypertension and congestive heart failure. Apart from the chemotherapy regimen, the patient was taking carvedilol, furosemide and ranitidine at home. The patient appeared comfortable but complained of poor appetite and weight loss. She denied any heartburn, dysphagia or odynophagia. Physical examination revealed a cachectic, euvolemic woman with palpebral conjunctival pallor and a midline surgical abdominal scar, but no obvious mucosal or skin lesions. The initial set of laboratory investigations revealed severe anemia, with hemoglobin level of $61 \mathrm{~g} / \mathrm{L}$, a white blood cell count of $16 \times 10^{9} / \mathrm{L}$ ( $88.8 \%$ segmented neutrophils, $6.3 \%$ lymphocytes, $4.4 \%$ monocytes), a platelet count of $250 \times 10^{9} / \mathrm{L}$ and renal dysfunction, with an elevated serum creatinine level of $3.5 \mathrm{~g} / \mathrm{L}$ and blood urea nitrogen level of $38 \mathrm{~g} / \mathrm{L}$.

Esophagogastroduodenoscopy (EGD) was performed and the images of the findings are shown in Figures 1 and 2. Biopsies obtained from the esophagus revealed squamous mucosa with ulceration and crystal deposits (Figures 3A and 3B).

\section{DISCUSSION}

Esophagitis dissecans superficialis secondary to 5-FU use EGD revealed severe desquamative esophagitis with no active bleeding (Figure 1). Violacaeous esophageal epithelium that instantly desquamated on contact with the flexible endoscope was noted (Figure 2). Esophageal biopsy revealed ulceration, cellular debris and crystalloid/foreign material (Figure 3A). Ballooning degeneration, edema, ulceration and cellular debris were observed at lower magnification (Figure 3B).

Esophagitis dissecans superficialis is a rare condition characterized by the sloughing of large sections of esophageal mucosa. Multiple conditions have been recognized as precipitants of this dramatic finding, including autoimmune bullous dermatoses such as pemphigus vulgaris or pemphigoid $(1,2)$; autoimmune enteropathy such as celiac disease (3); endoscopic esophageal manoeuvres, such as dilation (4), or variceal sclerotherapy (5); and medications such as bisphosphonates (6) or nonsteroidal anti-inflammatory drugs (7). This patient did not have any of these medical comorbidities and was not on any medication reported to cause this rare abnormality.

5 -FU is an antimetabolite and a pyrimidine analogue that irreversibly inhibits thymidylate synthase. Gastrointestinal tract mucositis is one of the most important dose-limiting side effects of 5-FU (8). Chemotherapy-induced generation of free radicals with DNA damage and subsequent initiation of inflammatory cytokine cascade with mucosal injury has been extensively studied $(9,10)$. The mucositis is an entity with a possible continuum of manifestations ranging from erythematous mucosa without a deficit in structural integrity, to florid desquamative lesions such as observed in our patient.

To our knowledge, the present case is the first report of an antimetabolite-induced esophagitis dissecans superficialis in the medical literature.

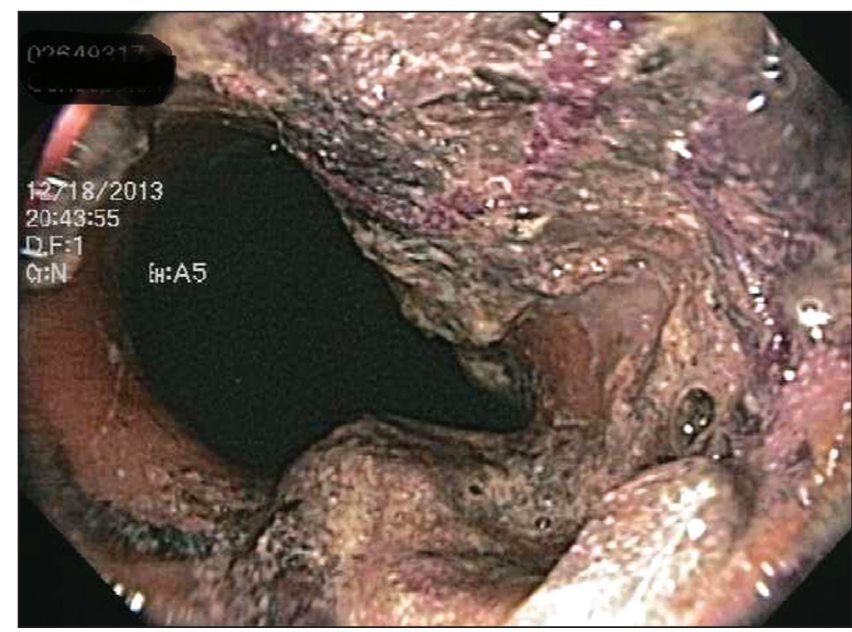

Figure 1) Severe desquamative esophagitis with no active bleeding on esophagogastroduodenoscopy

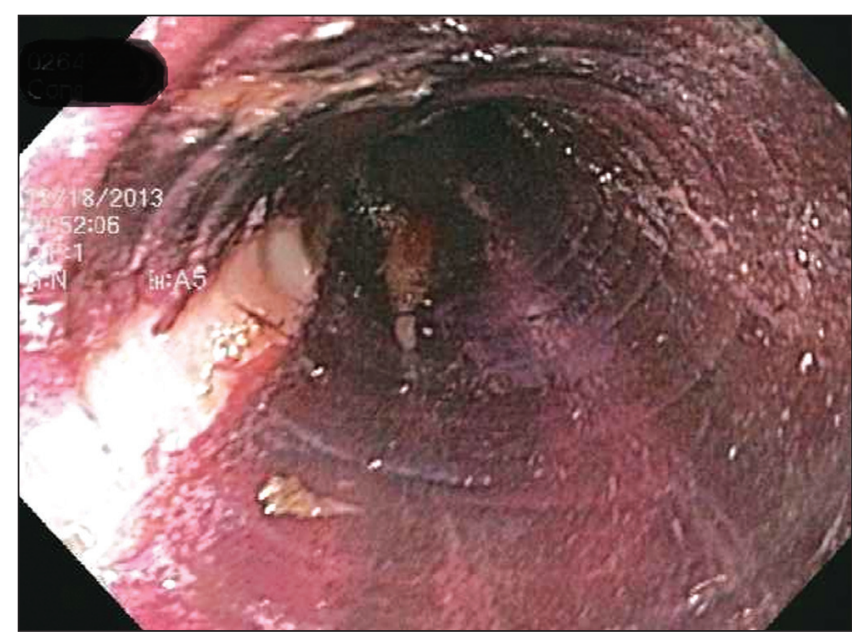

Figure 2) Violacaeous esophageal epithelium that instantly desquamated on contact with the flexible endoscope

Bronx Lebanon Hospital Center, Department of Medicine, Bronx, New York, USA

Correspondence: Dr Hassan Tariq, Bronx Lebanon Hospital Center, Department of Medicine, 1650 Selwyn Avenue, Suite 10C, Bronx,

New York 10457, USA. Telephone 718-960-1234, fax 718-960-2055, e-mail hassan663@hotmail.com

Received for publication January 6, 2015. Accepted January 12, 2015 


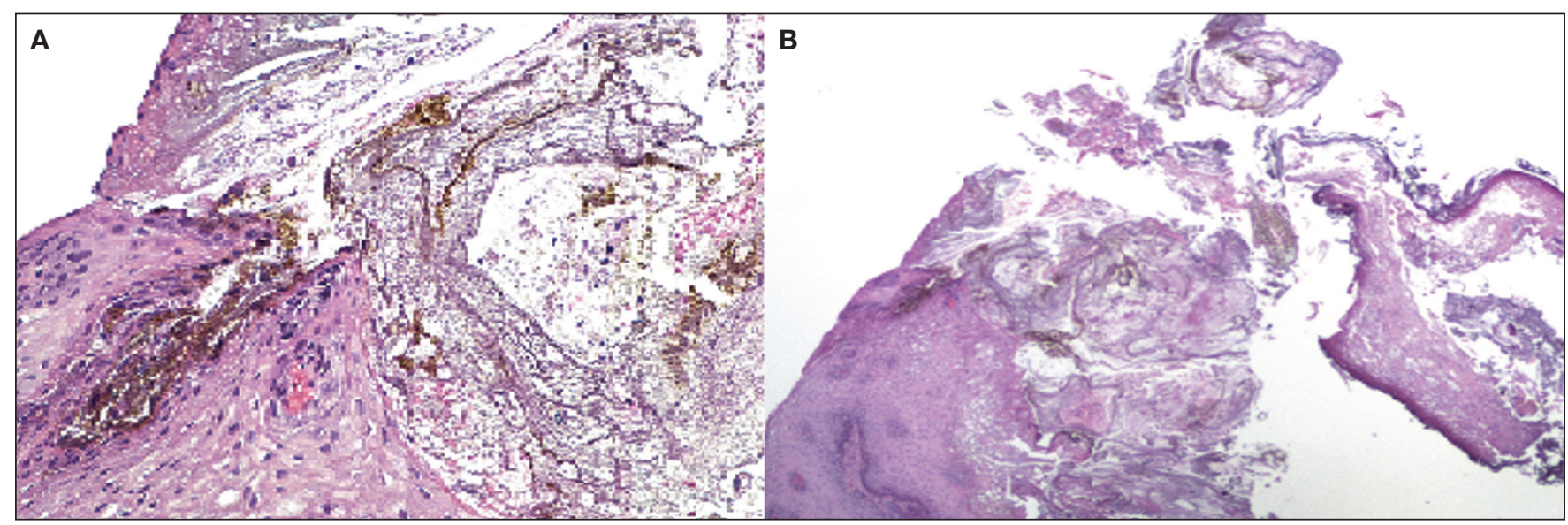

Figure 3) A Esophageal biopsy revealing ulceration, cellular debris and crystalloid/foreign material (original magnification $\times 100$ ). B Ballooning degeneration, edema, ulceration and cellular debris can be seen at lower magnification (original magnification $\times 40$ )

AUTHOR CONTRIBUTIONS: Vinaya Gaduputi MD: wrote the case presentation and was the endoscopist; Hassan Tariq MD: wrote the discussion, performed the literature search and reviewed the slides with the pathologist; Ariyo Ihimoyan MD: critical revision of the manuscript and the endoscopist.

\section{REFERENCES}

1. Hokama A. Esophagitis dissecans superficialis and autoimmune bullous dermatoses: A review. World J Gastrointest Endosc 2010;2:252-6.

2. Kaplan R. Esophagitis dissecans superficialis associated with pemphigus vulgaris. J Am Acad Dermatol 1981;4:682-7.

3. Hage-Nassar G. Esophagitis dissecans superficialis associated with celiac disease. Gastrointest Endosc 2003;57:140-1.

4. Ramaswami G. Oesophagitis dissecans superficialis complicating repeated rigid oesophagoscopy and dilatation. J Laryngol Otol 2007;121:92-3.

5. Pérez-Carreras M. Esophagitis dissecans superficialis (esophageal cast) complicating esophageal sclerotherapy. Am J Gastroenterol 1998;93:655-6.
DISCLOSURES: The authors have no financial disclosures or conflicts of interest to declare.

6. Hokama A. Esophagitis dissecans superficialis associated with bisphosphonates. Endoscopy 2007;(39 Suppl 1):E91.

7. Carmack S. Esophagitis dissecans superficialis ("sloughing esophagitis"): A clinicopathologic study of 12 cases.

Am J Surg Pathol 2009;33:1789-94.

8. Chang C. 5-Fluorouracil induced intestinal mucositis via nuclear factor- $\mathrm{kB}$ activation by transcriptomic analysis and in vivo bioluminescence imaging. PloS One 2012; 7:e31808.

9. Sonis ST. Pathobiology of oral mucositis: Novel insights and opportunities. J Support Oncol 2007;5(9 Suppl 4):3-11.

10. Al-Dasooqi N. Emerging evidence on the pathobiology of mucositis. Supportive care in cancer. 2013;21:3233-41. 


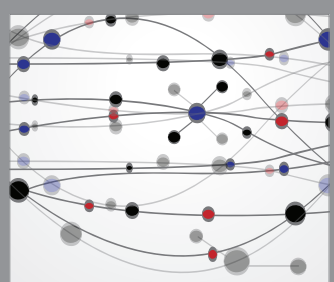

The Scientific World Journal
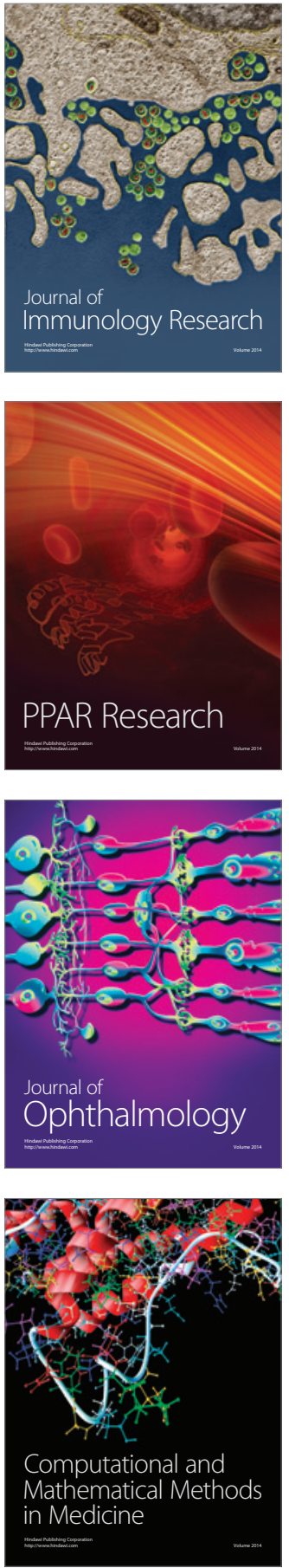

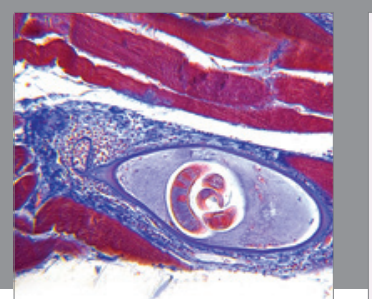

Gastroenterology Research and Practice

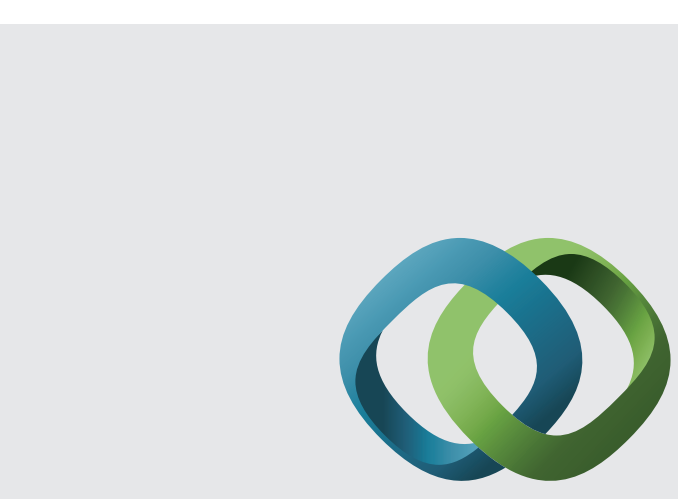

\section{Hindawi}

Submit your manuscripts at

http://www.hindawi.com
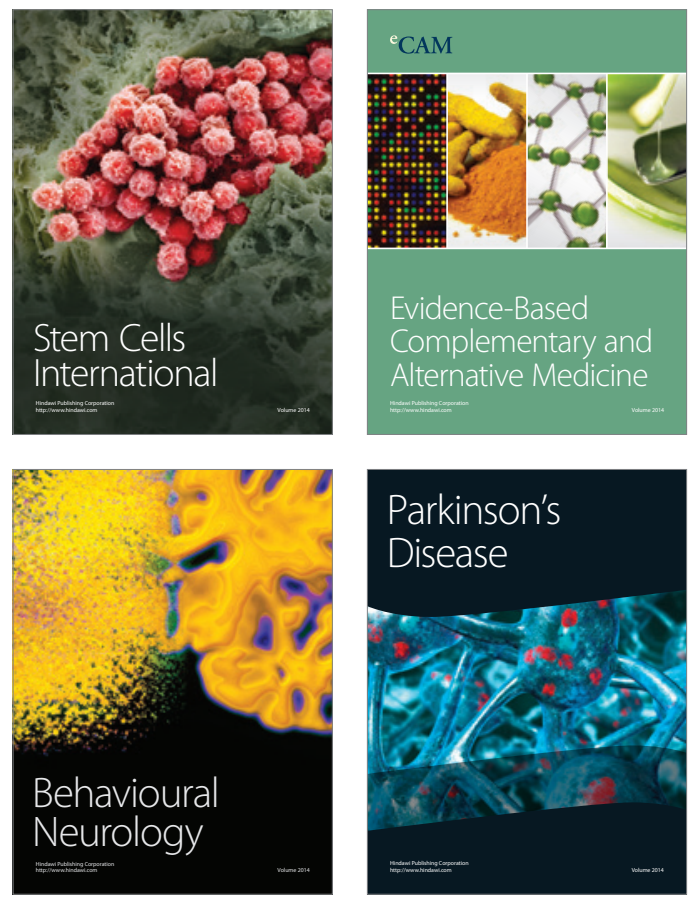
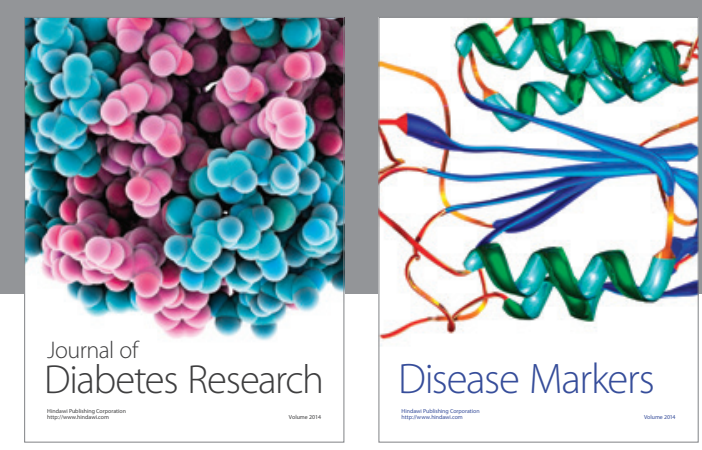

Disease Markers
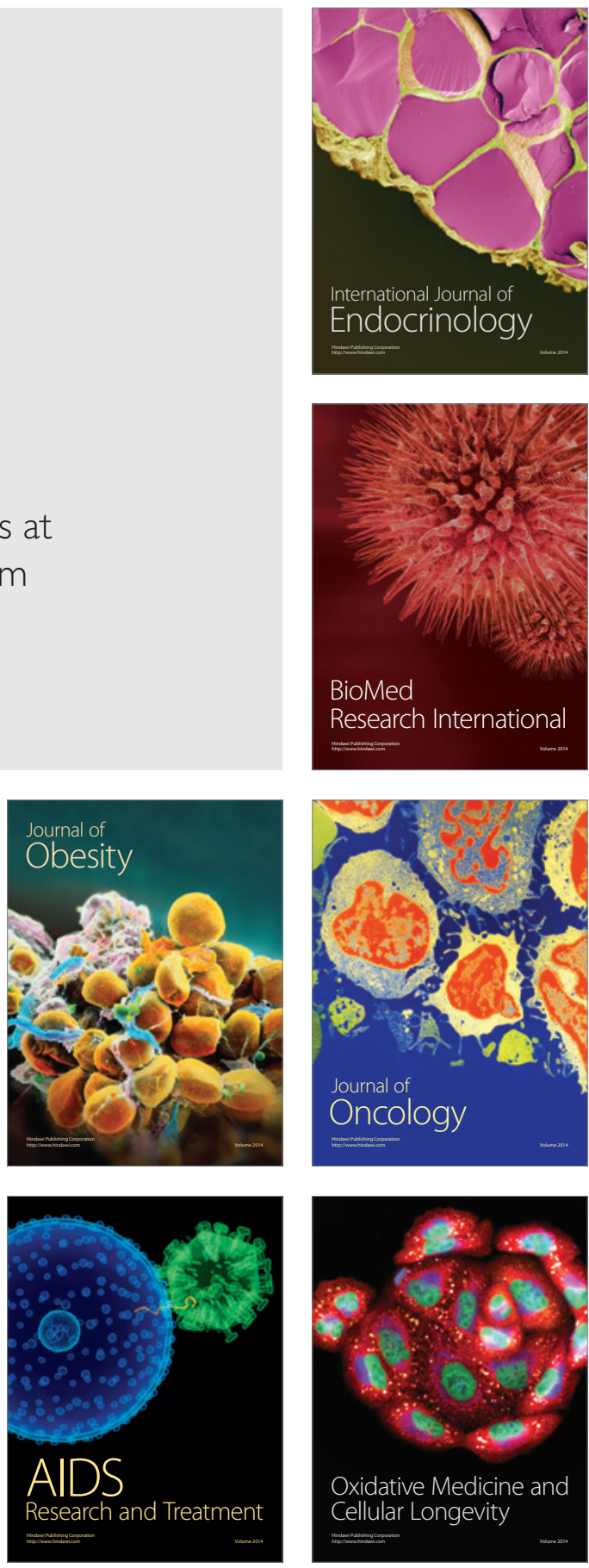